\title{
Etalonnage en force du Cavermod, moyen d'essai d'érosion de cavitation
}

\author{
par E.G. Filali, J.M. Michel et J.P. Franc \\ * L.E.G.I./i.m.g., \\ S. Hattori \\ ** Dept. of Mechanical Engineering, Fukui University
}

\section{INTRODUCTION}

Le Cavermod est un dispositif expérimental de table destiné à produire un vortex de vapeur et à le faire imploser violemment dans des conditions contrôlées de manière à produire des indentations permanentes d'érosion sur les matériaux solides. Effectivement, ce moyen s'avère extrêmement efficace du point de vue de l'érosion puisqu'un grand nombre d'indentations peuvent être obtenues sur un seul "tir" du dispositif, c'est-à-dire une seule implosion du tourbillon. Une première étude [1] a permis de cerner les caractères principaux de l'écoulement formé lors de l'effondrement du tourbillon de vapeur, de déterminer ses temps et vitesses caractéristiques (temps d'effondrement de l'ordre de la milliseconde, vitesses de l'ordre de plusieurs centaines de mètres par seconde) et de déterminer aussi la fenêtre temporelle et spatiale dans laquelle s'insère l'événement érosif. En même temps [2], la morphologie des indentations produites sur des métaux de duretés différentes a fait l'objet d'une première approche statistique et une modélisation numérique approfondie a permis d'évaluer les ordres de grandeur des pics de pression provoqués localement dans l'écoulement. La mesure effective des pics de pression produits sur les éprouvettes d'essais se heurte, comme en tout autre site érosif, aux difficultés bien connues que sont d'une part le très court temps de montée de l'impulsion, de l'ordre de la microseconde, et d'autre part la disproportion de taille entre les impacts de pression (de l'ordre du centième au dixième de millimètre carré) et les éléments sensibles des capteurs de pression (au moins le millimètre carré). Une autre difficulté résulte évidemment de l'agressivité du phénomène étudié lui-même.

Dans ces conditions, si l'on cherche à obtenir au moins une valeur approximative des pics de pression, on est conduit à dissocier l'évaluation de la taille des impacts érosifs de la mesure des forces elle-même. Cette méthode a déjà été utilisée au sein du LEGI par Franc et al [3]. La taille des impacts de pression est donnée par l'examen des indentations permanentes produites dans les premières phases de l'attaque érosive. La mesure des forces est faite à l'aide de capteurs spécifiques, dont les principes sont entièrement différents :
1. Capteurs constitués par des monocristaux d'Oxyde de Magnésium $(\mathrm{MgO})$ et développant sous choc des dislocations dont la taille peut être reliée à l'intensité du choc après étalonnage statique par essai de dureté. Cette technique a été développée par le Professeur Hattori de l'Université de Fukui.

2. Capteurs dont l'élément sensible, à base de céramique piézo-électrique, reçoit l'impulsion agressive reçue d'abord puis transmise par une éprouvette en cuivre, ce qui permet d'associer l'examen du signal électrique à celui des indentations d'érosion, tout en protégeant l'élément sensible. La conception et la réalisation de ces capteurs est due aussi au Professeur Hattori.

Une troisième méthode, développée au LEGI et utilisant des films PVDF [3] a été aussi utilisée, mais nous ne la présentons pas ici.

L'utilisation de capteurs à réponse électrique nécessite leur étalonnage préliminaire dans des conditions dynamiques. Un étalonnage dynamique idéal devrait se situer dans la même gamme de temps de montée de signal et d'amplitude que celle du phénomène étudié. Il est difficile de trouver un dispositif qui donne une force connue satisfaisant à ces deux conditions. Nous utilisons l'étalonnage par chute et rebond de bille dont le temps de montée est du même ordre de grandeur et qui de plus nous permet de comparer notre méthode à celle d'autres laboratoires, en particulier les laboratoires japonais.

On doit noter que l'approche du phénomène par mesure de force et non de pression est particulièrement indiquée dans notre cas. En effet, l'examen des figures d'érosion montre que la répartition de la pression à l'intérieur de la zone de choc (dont la taille est approximativement celle du cœur de vapeur initial) est très peu homogène, aussi bien dans l'espace que dans le temps : des pics de pression très localisés doivent se produire à des instants successifs en plusieurs points de cette zone. Dans ces conditions, on ne peut actuellement mesurer que l'intégrale de ces événements successifs. On peut cependant espérer que l'analyse du signal délivré par les capteurs à réponse électique permettra d'accéder indirectement à ces événements élémentaires. 


\section{II — DISPOSITIF EXPÉRIMENTAL}

L'élément principal du CAVERMOD est une chambre tournante, remplie de liquide désaéré, limitée latéralement par une surface de révolution et, sur ses extrémités, d'une part par une section droite fixe pouvant porter des capteurs de pression ou des éprouvettes d'érosion, d'autre part par un piston mobile (figure 1).

Dans un premier temps, la chambre tournant à une vitesse de rotation $\Omega$, le piston est déplacé d'une valeur a. L'augmentation de volume conduit à la formation d'un tourbillon de vapeur de rayon $\mathbf{r}_{0}$. Après un laps de temps suffisant pour permettre la stabilisation du tourbillon, le système de commande communique au piston un mouvement rapide de retour à sa position initiale, ce qui provoque l'implosion axiale du tourbillon à la vitesse $\mathbf{V}$.

Le dimensionnement du CAVERMOD résulte d'une modélisation simplifiée dans laquelle le piston joue le rôle d'un batteur à houle et la force centrifuge celui de l'accélération de la pesanteur. La longueur de la section d'essai est $\mathbf{L}=\mathbf{1 5 6} \mathbf{~ m m}$; le rayon du piston est $\mathbf{R}=\mathbf{2 1} \mathbf{m m}$. La taille initiale du tourbillon de vapeur est donnée par la conservation des volumes, soit $\mathbf{r}_{0}{ }^{2} / \mathbf{R}^{2}=\mathbf{a} / \mathbf{L}$. La condition d'implosion axiale nécessite une vitesse minimale de retour du piston à partir de laquelle la hauteur de l'intumescence est égale au rayon du tube de vapeur. Dans la pratique, cette valeur limite est assez petite (environ $0,32 \mathrm{~m} / \mathrm{s}$ lorsque la vitesse de rotation de la section d'essai est $\Omega=8000 \mathrm{tpm}$ ), de sorte que cette condition est facilement réalisée.

Le retour rapide du piston est obtenu par le choc d'une masselotte mise en mouvement dans un cylindre de longueur $40 \mathrm{~cm}$, par l'effet d'une différence de pression $\Delta \mathbf{P}$ (de l'ordre de 1 à 6 bars) sur ses deux faces. La vitesse d'implosion axiale $\mathbf{V}$ est alors de plusieurs centaines de mètres par
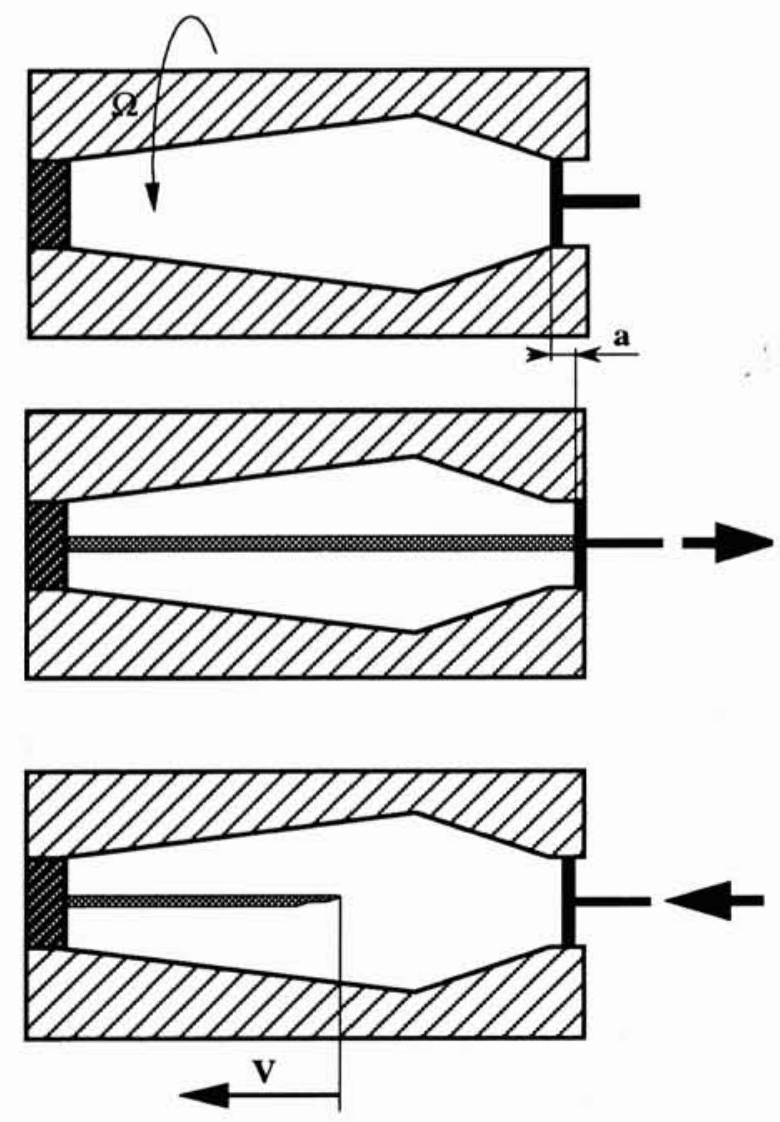

1. Principe du CAVERMOD. seconde. Elle augmente avec $\Delta \mathbf{P}$ et avec $1 / \mathbf{r}_{\mathrm{o}}{ }^{2}$. La surpression maximale obtenue sur l'éprouvette est de l'ordre de $\rho c V$ où $\rho c$ est l'impédance acoustique du liquide. Les paramètres qui déterminent l'écoulement, pour une géométrie fixée par $\mathbf{L}$ et $\mathbf{R}$, sont ainsi a (ou $\mathbf{r}_{\mathbf{o}}$ ), $\Omega$ et $\Delta \mathbf{P}$.

\section{E ÉTALONNAGE PAR MACRO-CRISTAUX D'OXYDE DE MAGNÉSIUM}

\subsection{Caractéristiques du matériau}

Le monocristal d'oxyde de magnésium $(\mathrm{MgO})$ utilisé dans cette étude est pur à $99,99 \%$. Ses caractéristiques mécaniques sont les suivantes : résistance à la flexion $772 \mathrm{mPa}$, module d'Young $251 \mathrm{GPa}$, cofficient de Poisson 0,25. C'est un matériau transparent, de haute dureté (dureté Vickers 866), à comportement fragile. Il a l'avantage de pouvoir être obtenu en macrocristaux facilement façonnables. Les expériences faites avec ce type de matériau permettent d'obtenir un système de glissements stables qui peuvent être reliés à l'intensité du choc reçu. Il révèle un système de dislocations à la surface ou dans la section transversale d'un échantillon soumis à une sollicitation donnée.

Le monocristal d'oxyde de magnésium a six plans de coupe, et six directions de coupe $\langle 110\rangle$ comme le montre la figure 2 . Dans la figure (2a), les quatre plans $\{110\}$ repérés par (110), (110), (101) et (101), coupent le niveau de surface (100) à 45 degrés. Les lignes de dislocation qui émergent en haut de la surface sont appelées "dislocations Vis" car elles restent parallèles à leur direction de glissement. Dans la figure (2b), un ensemble de deux plans \{011) notés par (011)et (011) coupent la surface à 90 degrés. Les dislocations sur ces plans restent perpendiculaires à leur direction de glissement et sont appelés "dislocations Coin". Le système de dislocations produit dans le $\mathrm{MgO}$ permet d'identifier la nature des forces exercées. Ainsi des forces normales donnent des dislocations de type Vis, et des forces tangentielles donnent des dislocations de type Coin.

Dans nos expériences, le cristal d'oxyde de magnésium est coupé selon la surface 001 en échantillons de dimensions (5x5) $\mathrm{mm}$ et $2 \mathrm{~mm}$ d'épaisseur qui sont collés sur l'éprouvette d'érosion du Cavermod. Les échantillons, soumis à des essais de dureté ou à des essais de cavitation, sont gravés, à température de chambre, dans une solution adaptée. Les déformations peuvent alors être observées à l'aide d'un microscope optique ou électronique.

\section{- 3.2 Résultats expérimentaux et discussion}

\subsection{1 Étalonnage du cristal d'oxyde de magnésium}

La figure 3 présente les déformations obtenues lors d'un essai de dureté Rockwell avec une bille de 1,6 mm de diamètre, sous une charge de $150 \mathrm{~N}$. Les lignes de dislocation autour d'une indentation contiennent des "dislocations Coin" dans les directions [011] et [011] ainsi que de "dislocations Vis" dans les directions [010] et [001]. La présence de "dislocations Vis et Coin" montre que lors d'un essai de dureté, les forces qui agissent à la surface de l'échantillon sont à la fois des forces verticales et horizontales. Il est intéressant de noter que l'utilisation de l'oxyde de magnésium met en évidence les déformations plastiques produites à la périphérie des indentations de surface.

Les essais de dureté Rockwell et Vickers réalisés sur des échantillons de $\mathrm{MgO}$ permettent de mesurer les longueurs des lignes de dislocations, en surface et dans la section transversale, et de les relier à l'intensité de la force appli- 


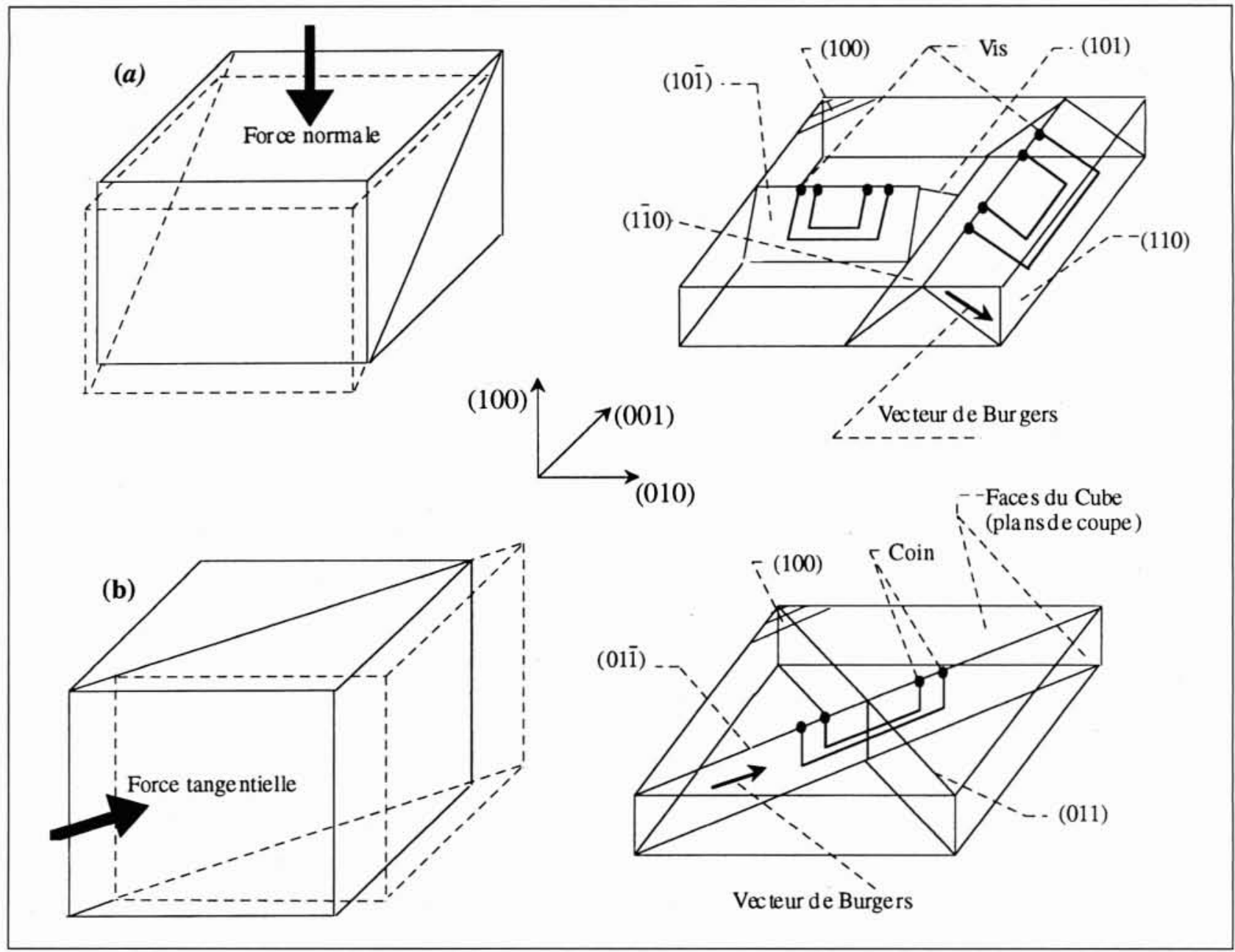

2. Systèmes de dislocations du cristal d'oxyde de magnésium ;

a. Plans de glissement (110) $45^{\circ}$

b. Plans de glissement (011) $90^{\circ}$

quée. Ainsi le graphe de la figure 5 montre une variation linéaire, dans un système d'échelle log-log, des longueurs des dislocations Vis en fonction de la charge appliquée. On en déduit une dépendance approximative de ces longueurs en $\mathrm{F}^{1 / 2}$. Chaque valeur représente la moyenne de cinq mesures, relevées en surface et dans la section transversale.

\subsection{2 Étalonnage du Cavermod}

La figure $4 \mathrm{a}$, prise au microscope optique, présente la surface (100) d'un échantillon de $\mathrm{MgO}$ ayant été exposé à l'implosion d'un tourbillon de vapeur. En plus des indentations présentes à la surface de l'échantillon, des déformations plastiques peuvent également être observées. Il s'agit d'un enchevêtrement de lignes de dislocations. Parfois ces lignes sont présentes sans les indentations. On remarque aussi la présence dominante des "dislocations Vis" et l'absence quasi totale des "dislocations Coin". Des observations similaires ont été faites par Hattori [4], qui a étudié le comportement plastique des cristaux de $\mathrm{MgO}$ soumis à des essais de cavitation dans un Venturi et un appareil d'essai vibratoire. Il observe que les dislocations se présentent uniquement sous forme de "dislocations Vis", et en conclut que les déformations plastiques occasionnées par la cavitation sont dues uniquement à des forces normales. Cette constatation semble corroborée par nos propres observations.

La mesure des longueurs des dislocations Vis s'effectue à la surface, ainsi que sur la section transversale (001) de l'échantillon, figure $4 \mathrm{~b}$. Une moyenne entre les deux valeurs permet alors de déterminer la force provoquée par l'implosion du tourbillon.

Les valeurs des longueurs des dislocations correspondant à des $\Delta \mathrm{P}$ croissants, ainsi que la valeur de la force d'impact correspondante, sont présentées dans le graphe de la figure 6 . Les valeurs de la force d'impact sont obtenues à partir du graphe 5. Remarquons que la force engendrée par l'implosion du tourbillon de vapeur est linéaire par rapport à la pression motrice produite par le choc du piston. La force d'impact varie de $20 \mathrm{~N}$ pour une pression motrice de $1 \mathrm{bar}$, à $150 \mathrm{~N}$ dans le cas d'une pression motrice de 4 bars, ce qui donne des pressions moyennes moins grandes que la valeur correspondant à la formule du coup de bélier lorsque l'aire de la surface attaquée est estimée à quelques millimètres carrés. 


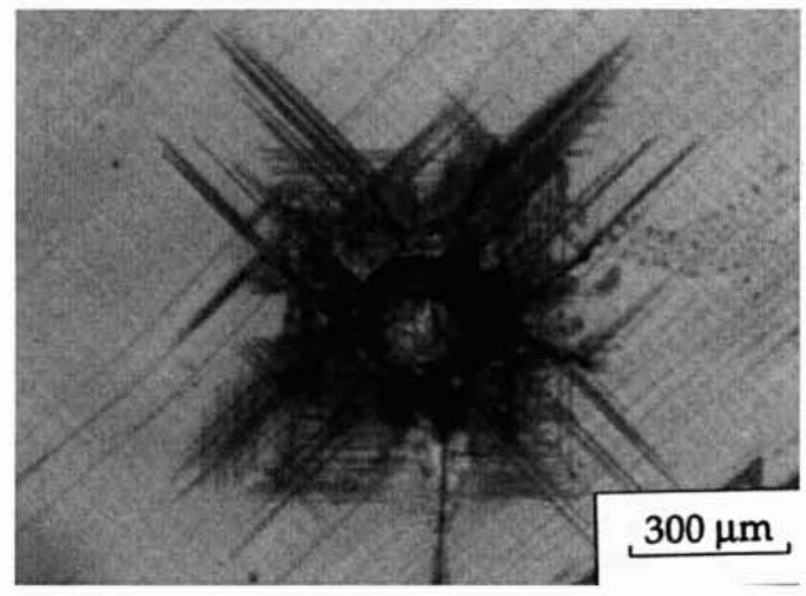

3. Dislocations produites sur les monocristaux d'oxyde de magnésium dans un essai de dureté Rockwell.

\section{EÉTALONNAGE PAR CAPTEUR DE PRESSION}

\section{- 4.1 Description du capteur}

Ces nouveaux capteurs de pression, utilisant des céramiques piézo-électriques, sont particulièrement développés par Hattori. Ils ont la particularité de pouvoir mesurer simultanément la force d'impact produite par l'implosion d'une structure de vapeur, ainsi que les dégâts érosifs qui y sont occasionnés. La surface de détection du capteur est, en effet, constituée de matériaux solides permettant le repérage et le comptage des indentations. Dans nos expériences, nous avons utilisé des capteurs en cuivre ayant des diamètre de 1 , 3 et $5 \mathrm{~mm}$. La figure 7 présente la schéma d'un de ces capteurs. Après fabrication, les capteurs sont montés sur des porte-échantillons adaptés.

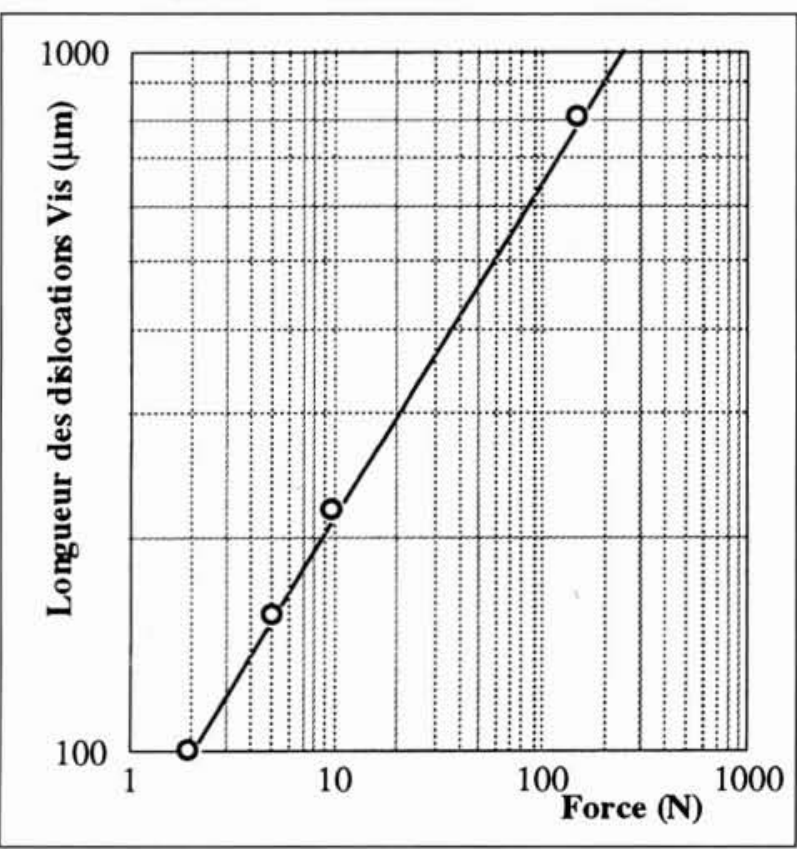

5. Etalonnage des monocristaux de $(\mathrm{MgO})$
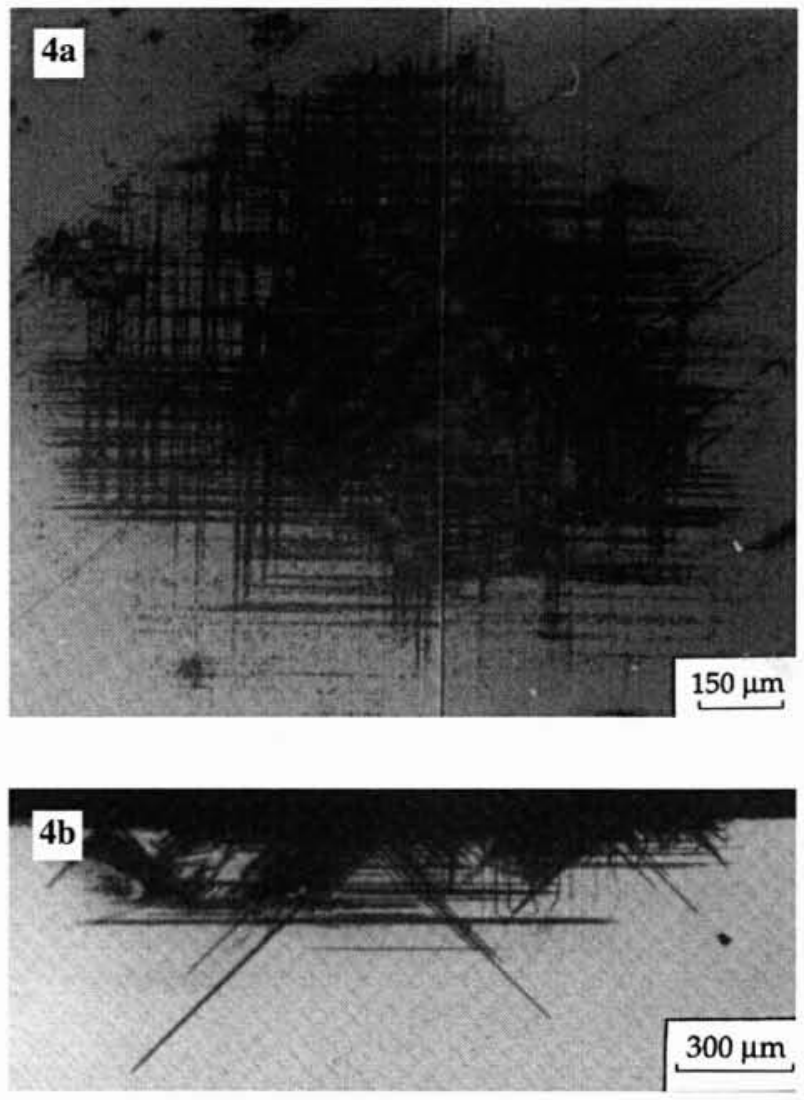

4. Dislocations de MgO produites par la Cavermod; a. en surface ( $\Delta \mathrm{P}=3,0$ bars, $\mathrm{a}=0,5 \mathrm{~mm}, \Omega=8000 \mathrm{tpm})$

b. coupe transversale $(\Delta \mathrm{P}=2,0$ bars, $\mathrm{a}=0,5 \mathrm{~mm}$, $\Omega=8000 \mathrm{tpm})$.

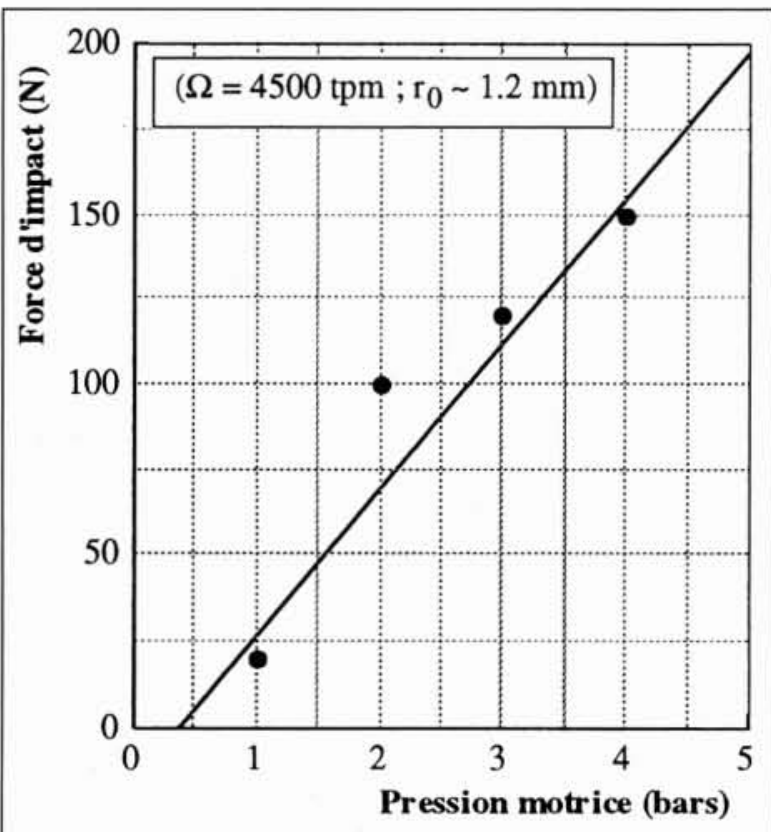

6. Etalonnage du Cavermod avec les monocristaux de (MgO) 


\section{2 Étalonnage du capteur}

Comme la sensibilité du capteur est affectée par les conditions de contact du capteur avec le porte-échantillon (dans notre cas, nous avons opté pour un collage légèrement élastique), le capteur est étalonné, par chute de bille, après avoir été monté. L'avantage de ce système d'étalonnage est qu'il est simple à réaliser et permet d'obtenir des forces importantes (de l'ordre de quelques Newtons), présentant une durée relativement courte (quelques microsecondes). Les conditions d'étalonnage ne sont donc pas trop éloignées de celles des mesures.

La force est calculée par la loi de la dynamique :

$\mathbf{F}=\mathrm{m} \cdot(1+\mathrm{e}) \cdot \mathrm{V}_{0} / \Delta \mathrm{t}$

où $\mathrm{m}$ est la masse de la bille, e est le coefficient de rebond (dans le cas du cuivre, $\mathrm{e}=0,55$ ), $\Delta \mathrm{t}$ est la durée du choc, $\mathrm{V}_{\mathrm{o}}$ est la vitesse d'impact déterminée à partir de la hauteur de chute de la bille.

Le coefficient e est déterminé par mesure de la hauteur de rebond, visualisées à l'aide d'une caméra rapide. Il dépend de la nature du matériau de détection ainsi que des conditions de collage du capteur.

Utilisant les valeurs mesurées, la relation entre la force et la tension de sortie est la suivante :

$\mathbf{F}($ Newton $)=2,95 \times \mathbf{V}$ (Volt)

Notons que le signal de sortie atteint couramment une amplitude de plusieurs dizaines de volts.

\section{- 4.3 Étalonnage du Cavermod}

La mesure des tensions délivrées par les capteurs (figure 8), permet à l'aide de la relation d'étalonnage des capteurs, de déterminer la force d'impact qui agit lors de l'implosion du tourbillon cavitant formé dans le Cavermod.

Les mesures de pression sont réalisées pour différentes valeurs de la pression motrice. Tous les essais sont réalisés avec une vitesse de rotation de $4500 \mathrm{tpm}$, valeur maximale autorisée par le fonctionnement du contact tournant. La taille du tourbillon est adaptée à cette vitesse de rotation. On relève dans chaque essai la valeur maximale du signal de sortie du capteur.

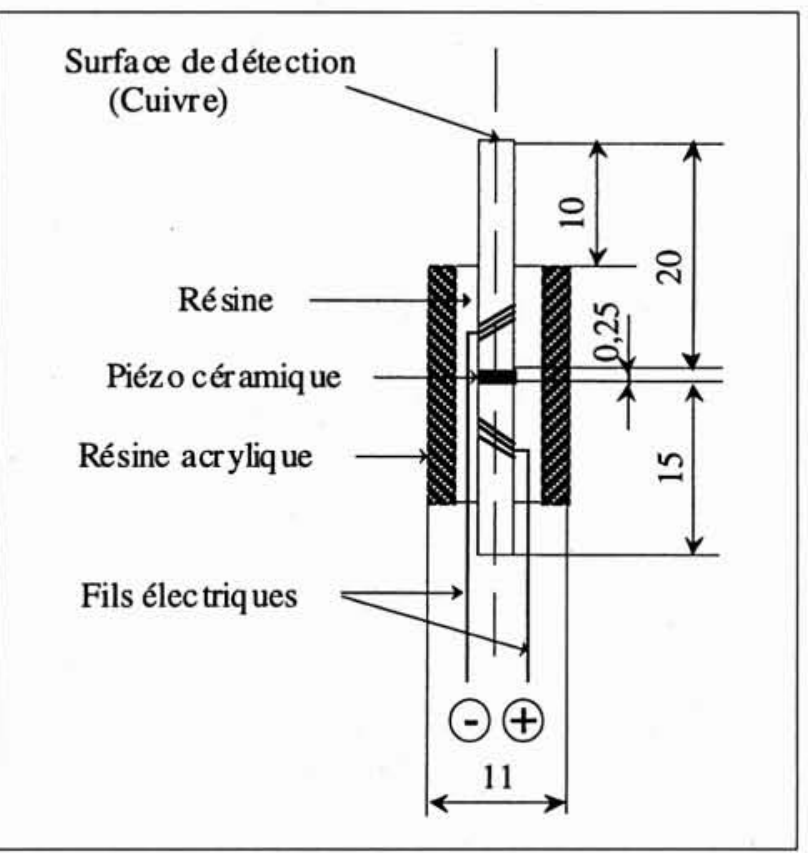

7. Capteur pression-érosion.
Les résultats obtenus sont présentés dans le graphe 9 ; les valeurs de force correspondant à chaque pression motrice sont le résultat d'une moyenne de 10 mesures. De la même manière que pour l'étude de l'oxyde de magnésium, la relation entre la force d'impact et la pression motrice est traduite par une variation sensiblement linéaire. Remarquons qu'il existe un seuil de pression motrice au dessous duquel le collapse ne produit pas de force d'impact détectable. On retrouve approximativement le même seuil avec les capteurs d'oxyde de magnésium.

Il est intéressant de noter que les deux méthodes d'étalonnage, quoique totalement différentes en principe, donnent sensiblement les même résultats.

\section{V — CARACTÉRISTIQUES DYNAMIQUES DU CAVERMOD}

Afin de qualifier certains aspects du Cavermod, nous avons effectué de nombreuses mesures avec les capteurs de pression en céramique pour étudier, par exemple, l'effet des variations

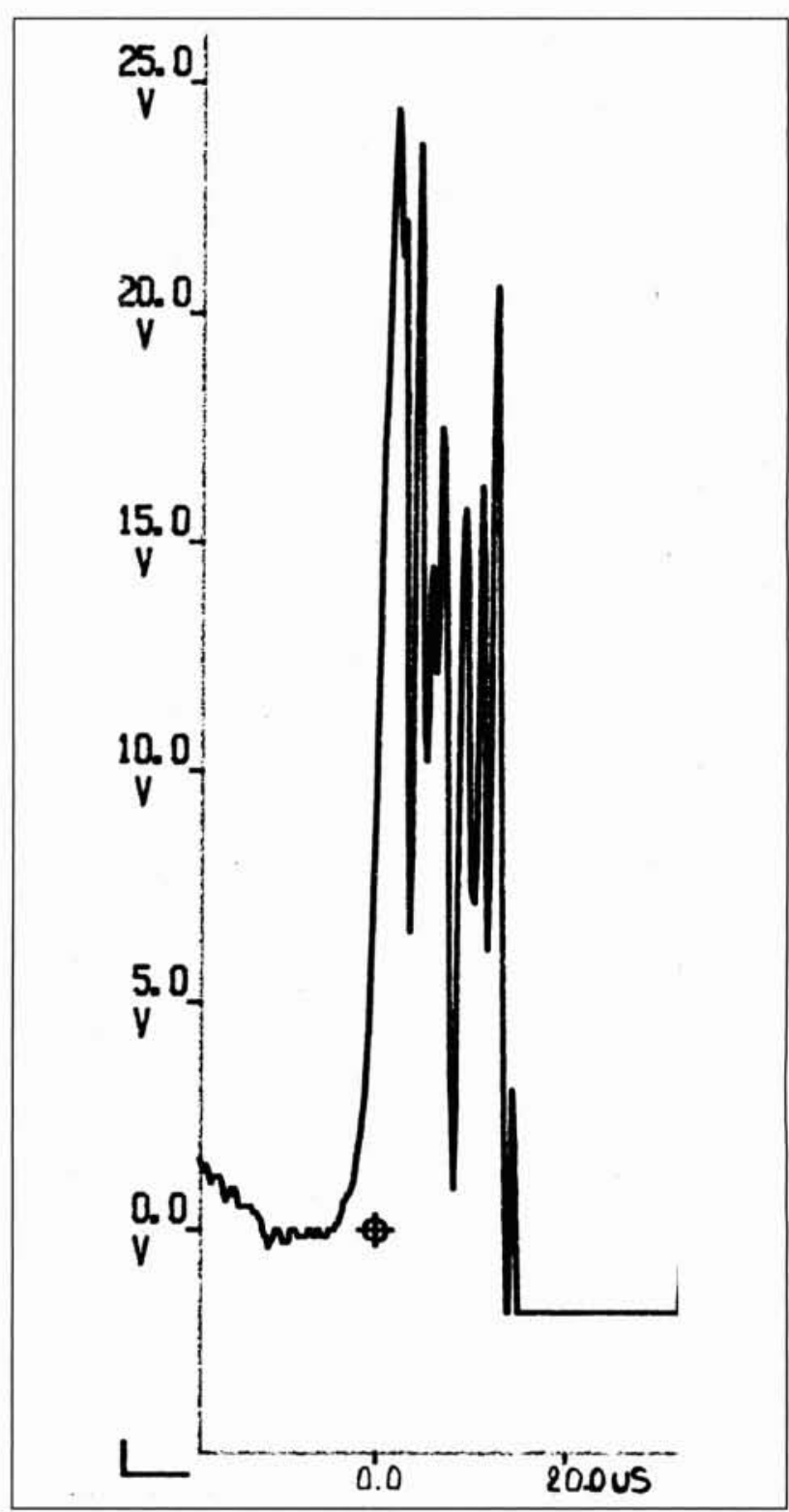

8. Signal du capteur. 


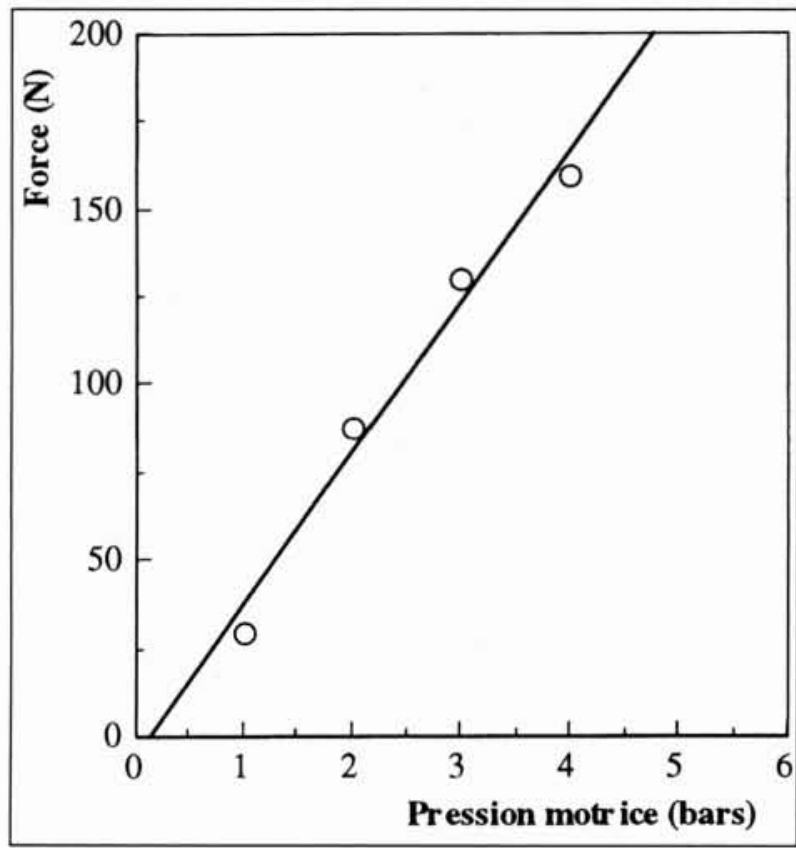

9. Etalonnage du Cavermod par mesure de pression.

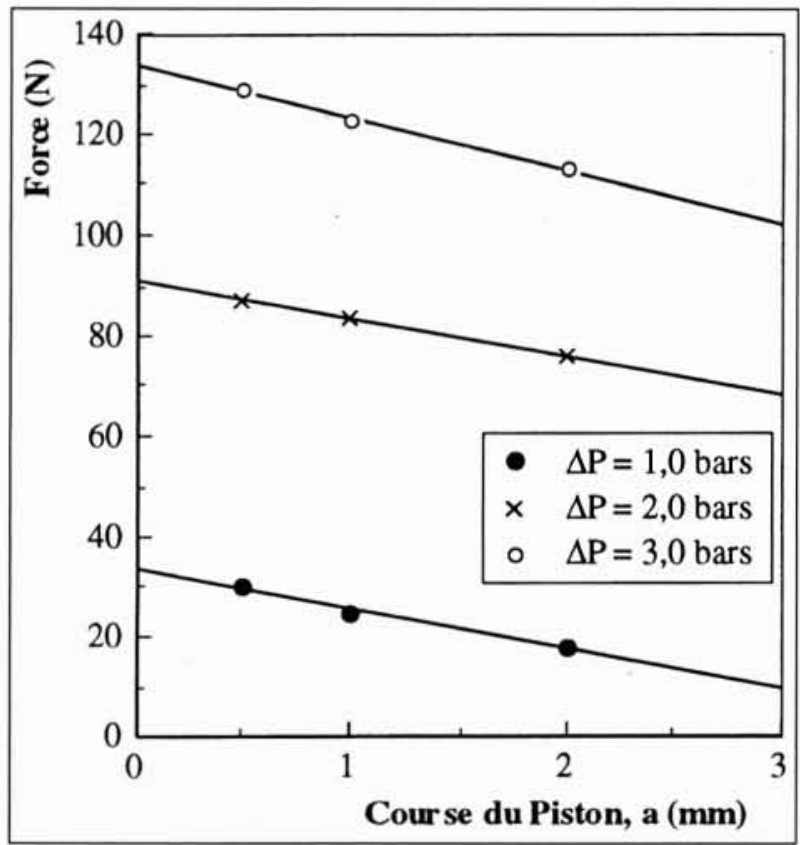

11. Effets des variations de la pression motrice.

du diamètre du capteur de pression et du diamètre du tourbillon, les autres paramètres étant gardés constants. Les résultats sont donnés respectivement dans les graphes 10 et 11 . Ces résultats montrent, entre autres, le caractère assez reproductible du Cavermod.

Le graphe 10 montre que lorsque la surface de détection du capteur varie, la force d'impact ne varie pas dans la même proportion, ce qui reflète la plus grande valeur de la pression au centre. Le graphe 11 ne fait que confirmer les premières constatations [2], [5] à savoir que lorsque le diamètre du tourbillon est petit, il y a une concentration et une

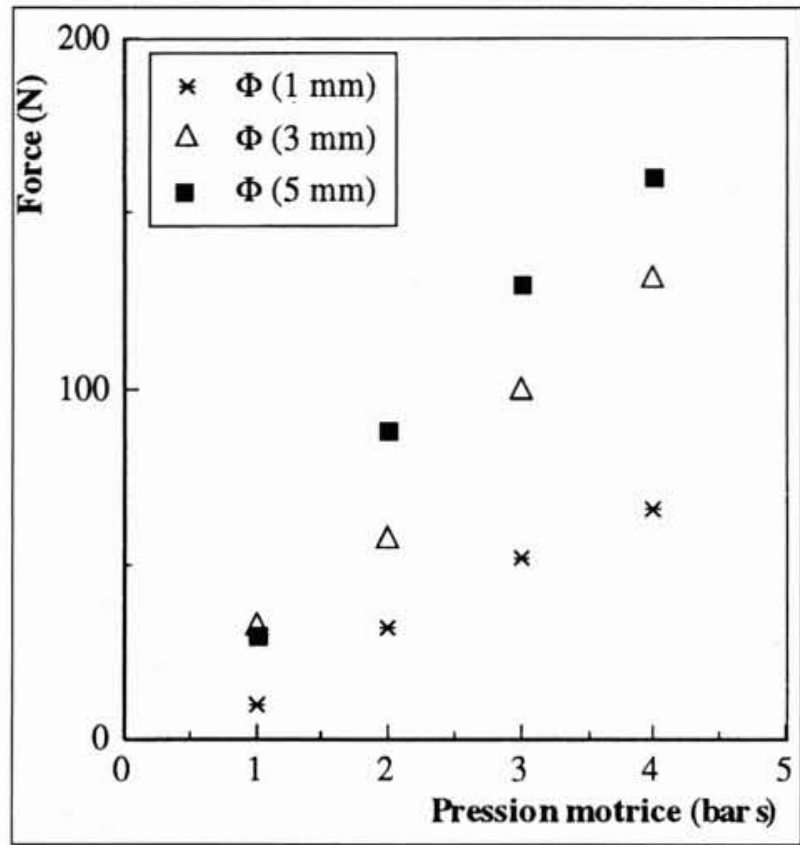

10. Effets des variations du diamètre du capteur.

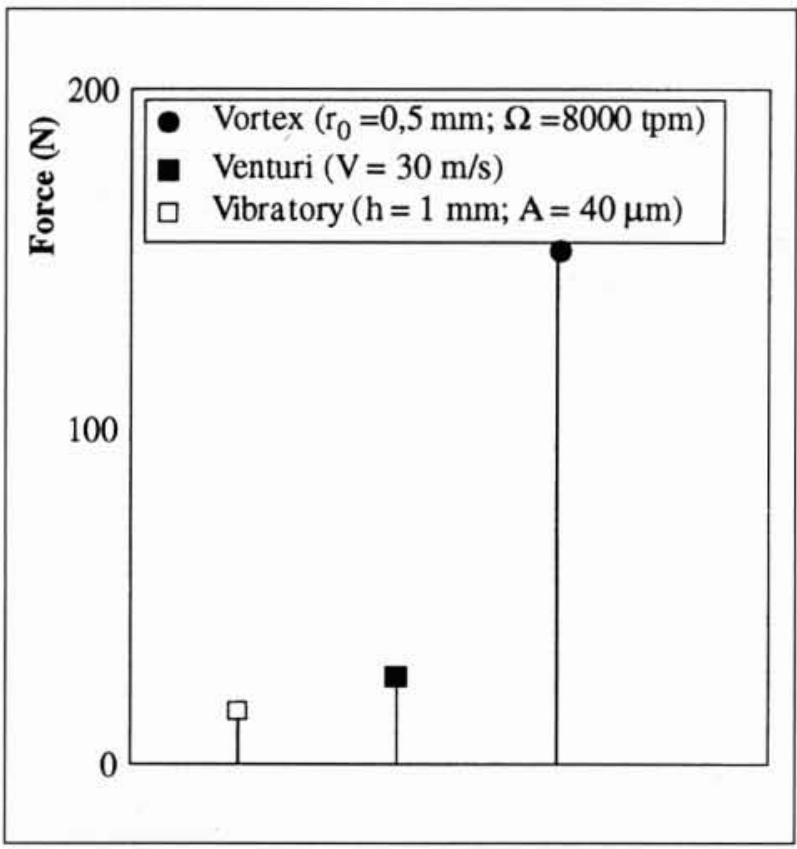

12. Capacité érosive du Cavermod.

focalisation de l'énergie sur une surface plus petite, et par conséquent, les forces engendrées sont plus importantes.

D'autres expériences ont été réalisées avec les cristaux d'oxyde de magnésium ; elles permettent de comparer la capacité érosive du Cavermod à celles d'autres moyens d'essai d'érosion de cavitation. Par exemple, le graphe 12 montre l'importance des dégâts que peut occasionner le collapse unique du tourbillon de vapeur produit dans le Cavermod, comparé à ceux de l'implosion de bulles cavitantes produites dans un écoulement de venturi ou dans le dispositif vibratoire normalisé de l'A.S.M.E. 


\section{VI $\mathbf{~ C O N C L U S I O N}$}

Le CAVERMOD est un dispositif entièrement original destiné à simuler des tourbillons cavitants dont l'efficacité agressive dans les machines hydrauliques a été remarquée depuis plusieurs années. La présente étude a permis d'étalonner le Cavermod (relation entre la pression motrice et la force du choc). Les grandes valeurs de force obtenues permettent d'expliquer l'efficacité érosive de ce dispositif, en comparaison avec celle d'autres dispositifs classiques. L'étape ultérieure de la recherche, déjà amorcée, consistera à corréler les pics du signal de sortie des capteurs aux indentations d'érosion : quelques résultats prometteurs ont déjà été obtenus dans ce sens [6].

\section{REMARQUE FINALE}

Lors des journées SHF des 13-14 novembre 1996, la présentation orale de cette contribution a fait état des derniers résultats obtenus, notamment la possibilité, en modifiant la forme du piston, d'obtenir un tourbillon plus court ce qui entraîne un changement de la figure d'érosion, réduite dans ce cas à un seul gros trou. Les discussions reflètent cette nouvelle donnée.

\section{RÉFÉRENCES}

[1] M.A. Dominguez-Cortazar, J.P. Franc, J.M. Michel. "The erosive axial collapse of a cavitating vortex : an experimental study". Article accepté au J. of Fluids Engineering.

[2] M.A. Dominguez-Cortazar "Le Cavermod, modèle physique de l'érosion de cavitation : qualification expérimentale et numérique" Thèse U.J.F., 22 Juillet 1994, Grenoble.

[3] J.P. Franc, J.M. Michel, H.N. Nguyen, A. Karimi "From pressure pulses measurements to mass loss prediction : the analysis of a method" 2nd International Symposium on Cavitation, Tokyo, April 1994.

[4] S. Hattori, K. Miyoshi, D.H. Buckley, T, Okada "Plastic Deformation of a Magnesium oxide (001) Surface Produced by Cavitation". J.of the Society of Tribologists and Lubrication Engineers. May 29, 1986.

[5] E.G. FIl.ALI, J.M. MiChEL "Effondrement d'un vortex tourbillonnaire: Visualisation de l'Implosion et Analyse des Dégâts Érosifs", Congrès de Mécanique. Constantine, 4-6 Décembre 1995.

[6] E.G. Filali, S. Hattori, J.M. Michel. "The Cavermod device : some recent results concerning its force calibration". French-Japanese Workshop on Cavitation Erosion. Grenoble, March 13-15, 1996. 